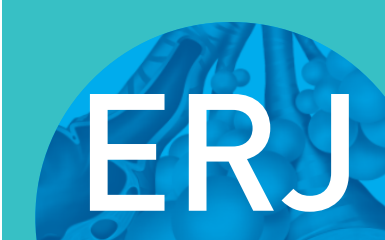

open research

\title{
The impact of perceived risk, screening eligibility and worry on preference for lung cancer screening: a cross-sectional survey
}

\author{
Katharine See ${ }^{1}$, Renee Manser ${ }^{2,3,4}$, Elyse R. Park ${ }^{5}$, Daniel Steinfort ${ }^{2}$, \\ Bridget King ${ }^{2}$, Francesco Piccolo ${ }^{6}$ and David Manners ${ }^{6,7}$
}

Affiliations: ${ }^{1}$ Dept of Respiratory Medicine, Northern Hospital, Epping, Victoria, Australia. ${ }^{2}$ Dept of Respiratory and Sleep Medicine, Royal Melbourne Hospital, Parkville, Victoria, Australia. ${ }^{3}$ Dept of Medicine (Royal Melbourne Hospital), University of Melbourne, Victoria, Australia. ${ }^{4}$ Dept of Medical Oncology and Haematology, Peter MacCallum Cancer Centre, Parkville, Victoria, Australia. ${ }^{5}$ Harvard Medical School, Massachusetts General Hosptial, Boston, MA, USA. ${ }^{6}$ Midland Physician Service, St John of God Midland Private and Public Hospitals, Midland, Western Australia, Australia. ${ }^{7}$ Curtin Medical School, Curtin University, Bentley, Western Australia, Australia.

Correspondence: David Manners, Midland Physician Service, St John of God Midland Public and Private Hospitals, 1 Clayton Rd, Midland, Australia 6056. E-mail: david.mannersdasjog.org.au

ABSTRACT Lung cancer screening is effective at reducing lung cancer deaths when individuals at greatest risk are screened. Recruitment initiatives target all current and former smokers, of whom only some are eligible for screening, potentially leading to discordance between screening preference and eligibility in ineligible individuals. The objective of the present study was to identify factors associated with preference for screening among ever-smokers.

Ever-smokers aged 55-80 years attending outpatient clinics at three Australian hospitals were invited. The survey recorded: 1) demographics; 2) objective lung cancer risk and screening eligibility using the Prostate Lung Colon Ovarian 2012 risk model; and 3) perceived lung cancer risk, worry about and seriousness of lung cancer using a validated questionnaire. Multivariable ordinal logistic regression identified predictors of screening preference.

The survey was completed by 283 individuals (response rate 27\%). Preference for screening was high (72\%) with no significant difference between low-dose computed tomography screening-eligible and -ineligible individuals ( $77 \%$ versus $68 \%, \mathrm{p}=0.11)$. Worry about lung cancer (adjusted-proportional odds ratio (adj-OR) 1.31, 95\% CI 1.08-1.58; $\mathrm{p}=0.007$ ) and perceived seriousness of lung cancer (adj-OR 1.31, 95\% CI 1.05-1.64; $\mathrm{p}=0.02$ ) were associated with higher preference for lung cancer screening while screening eligibility was not. The concept of "early detection" was the most important driver to have screening while practical obstacles like difficulty travelling to the scan or taking time off work were the least important barriers to screening.

Most current or former smokers prefer to undergo screening. Worry about lung cancer and perceived seriousness of the diagnosis are more important drivers for screening preference than eligibility status.

@ERSpublications

Preference for lung cancer screening is high among Australian current and former smokers. Worry about lung cancer and perceived seriousness of the disease are associated with screening preference, while eligibility based on lung cancer risk is not. http://bit.ly/36PZlYA

Cite this article as: See $\mathrm{K}$, Manser $\mathrm{R}$, Park ER, et al. The impact of perceived risk, screening eligibility and worry on preference for lung cancer screening: a cross-sectional survey. ERJ Open Res 2020; 6: 00158-2019 [https://doi.org/10.1183/23120541.00158-2019].

This article has supplementary material available from openres.ersjournals.com.

Received: 20 June 2019 | Accepted after revision: 23 Jan 2020

Copyright $\odot$ ERS 2020. This article is open access and distributed under the terms of the Creative Commons Attribution Non-Commercial Licence 4.0. 


\section{Introduction}

Implementation of lung cancer screening with low-dose computed tomography (LDCT) is challenging for many reasons. Unlike other established population-based cancer screening programmes that estimate risk and determine eligibility by readily available demographics such as age and sex alone, the eligibility assessment for lung cancer screening is more complex and includes, at a minimum, smoking history, which is not remotely available [1,2]. Recruitment initiatives will target all current and former smokers (ever-smokers), and may potentially lead to discordance between screening preference and eligibility in lower-risk individuals who smoked lightly or quit in the distant past. Such difficulties have been demonstrated in the real-world application of lung cancer screening with low uptake among eligible individuals, inappropriate use in low-risk individuals and high rates of ad hoc screening where screening is not currently recommended [3-5]. While LDCT screening is recommended and funded in the USA and other jurisdictions, it is not recommended or funded in Australia [1, 6, 7].

It is unclear whether an individual's objective risk of lung cancer, and therefore their screening eligibility, is correlated with their perceived risk of lung cancer or preference for undergoing screening [8-10]. While previous studies suggest that many at-risk individuals are supportive of screening, validated measures of risk perception have not been used to examine the association between screening preference and perceived risk, screening eligibility, and perceived seriousness of and worry about lung cancer. Thus, the aims of the study were to gauge the preference for lung cancer screening among Australian ever-smokers, identify any association between perceived lung cancer risk and LDCT screening eligibility on screening preference, and assess relative importance of possible screening drivers and barriers.

\section{Methods}

\section{Study design}

From January 2017 to July 2017, we conducted a cross-sectional written survey of 55-80-year-old current or former smokers attending respiratory and general medical outpatient clinics at three Australian hospitals. Clinical administrative staff opportunistically provided surveys to individuals attending the clinics who then completed the survey unassisted. The survey (supplementary material) recorded participant demographics, objective lung cancer risk and screening eligibility, perceived lung cancer risk, and worry about lung cancer. Participants then reviewed a short description of LDCT lung cancer screening, including benefits and harms, then provided their preference for screening assessed on a five-point Likert scale from "strongly agree" to "strongly disagree" to the statement "Overall, I would have lung cancer screening if it was offered to me". Preference for screening was defined as either "strongly agree" or "agree" to the previous statement. Objective lung cancer risk was determined using the Prostate Lung Colon Ovarian 2012 risk prediction model $\left(\mathrm{PLCO}_{\mathrm{m} 2012}\right)$ and "LDCT screening-eligible" was defined as a $\mathrm{PLCO}_{\mathrm{m} 2012}$ 6-year lung cancer risk of $>1.5 \%$ [11]. This threshold was more accurate than US Preventive Services Task Force criteria at predicting 6-year lung cancer incidence in a large Australian external validation cohort and is estimated to reduce the number needed to screen to prevent one death from 320 to $255[2,11]$. The participants were not aware of their eligibility status at the time. The risk perception elements of the survey, including worry about lung cancer and perceived seriousness of a lung cancer diagnosis, were developed and validated by PARK et al.[10] within a subset of National Lung Screening Trial participants. The risk perception component includes five questions with five-point Likert responses. Worry about lung cancer included combined responses to two four-point Likert questions on the frequency and intensity of lung cancer worry and perceived seriousness was assessed on a five-point Likert scale responding to "How serious would the health consequences be if you developed lung cancer?". These elements incorporated key theoretical cognitive and emotional components of the Health Belief Model, the Precaution Adoption Process Model and the Self-Regulation Model, which have been shown to be stable across time among screening participants. Questions evaluating potential drivers and barriers to screening were developed and piloted by the study team and assessed on a 10-point Likert scale. The study duration, limited by feasibility of running the study during outpatient clinics, determined the sample size. The study was approved by the Melbourne Health and St John of God Healthcare Human Research and Ethics Committees (QA2016167 and \#1073). Consent was implied by completion of the questionnaire.

\section{Statistical analysis}

Baseline characteristics are expressed as frequencies and percentages, means and standard deviations, or medians and interquartile ranges (IQRs). Group comparisons between patients who preferred and did not prefer screening were performed using the Chi-squared test, t-test or Wilcoxon Signed Rank test, as dictated by the data type. Correlation between $\mathrm{PLCO}_{\mathrm{m} 2012}$ lung cancer risk and lung cancer risk perception was assessed using Pearson's correlation. Factors associated with screening preference were identified using a multivariable ordinal logistic regression model that included all variables except potential drivers and barriers to screening, with a p-value $<0.2$ on univariate analysis as well as those plausibly linked to the 
outcome of interest. A p-value $<0.05$ was considered significant. Differences between drivers and barriers to screening were assessed with multiple per-respondent univariate comparisons using the Wilcoxon signed rank test with a Bonferroni-corrected significant p-value. Missing data were not imputed. Analysis was performed using STATA version 14.0 (StataCorp, College Station, TX, USA).

\section{Results}

760 individuals participated of a potential 2827 individuals aged 55-80 years who attended the clinics, yielding a response rate of $27 \%$. Of these, 455 were never-smokers and 23 ever-smokers did not provide sufficient information to determine their $\mathrm{PLCO}_{\mathrm{m} 2012}$ lung cancer risk and/or screening preference, leaving 283 included in the analysis. Demographic characteristics of study participants are detailed in table 1 . The mean age was $66.3 \pm 6.5$ years. $60 \%$ of participants were male, $92 \%$ were white and $79 \%$ were former smokers. There were no significant differences in baseline characteristics between participants who did and did not prefer screening.

The overall preference for participation in screening was high, with 204 (72\%) of the 283 either agreeing or strongly agreeing that they would undergo screening if offered. The median PLCO $_{\mathrm{m} 2012}$ lung cancer risk was 1.28\% (IQR $0.44-3.11 \%$ ); $45.6 \%$ of participants were eligible for screening. An individual's actual lung cancer risk was weakly correlated with perceived lung cancer risk $(\mathrm{r}=0.28, \mathrm{p}<0.0001)$. There was no significant difference in preference for participation in screening between the screening eligible and ineligible groups ( $77 \%$ versus $68 \%, \mathrm{p}=0.11)$ or between current versus former smokers $(68 \%$ versus $73 \%, \mathrm{p}=0.18)$.

The final multivariable screening preference model included higher education, smoking status, history of COPD, patient perception of risk, seriousness, worry and screening eligibility. Worry about lung cancer (adjusted-proportional odds ratio (adj-OR) 1.31, 95\% 1.08-1.58; $\mathrm{p}=0.007$ ) and perceived seriousness of lung cancer (adj-OR 1.31, 95\% CI 1.05-1.64; $\mathrm{p}=0.02$ ) were associated with preference for lung cancer screening (figure 1).

Among the three potential drivers for undergoing screening, the concept of early detection was rated more important than both reduced lung cancer mortality and reassurance/peace of mind that the person does not

\section{TABLE 1 Participant demographics and baseline characteristics}

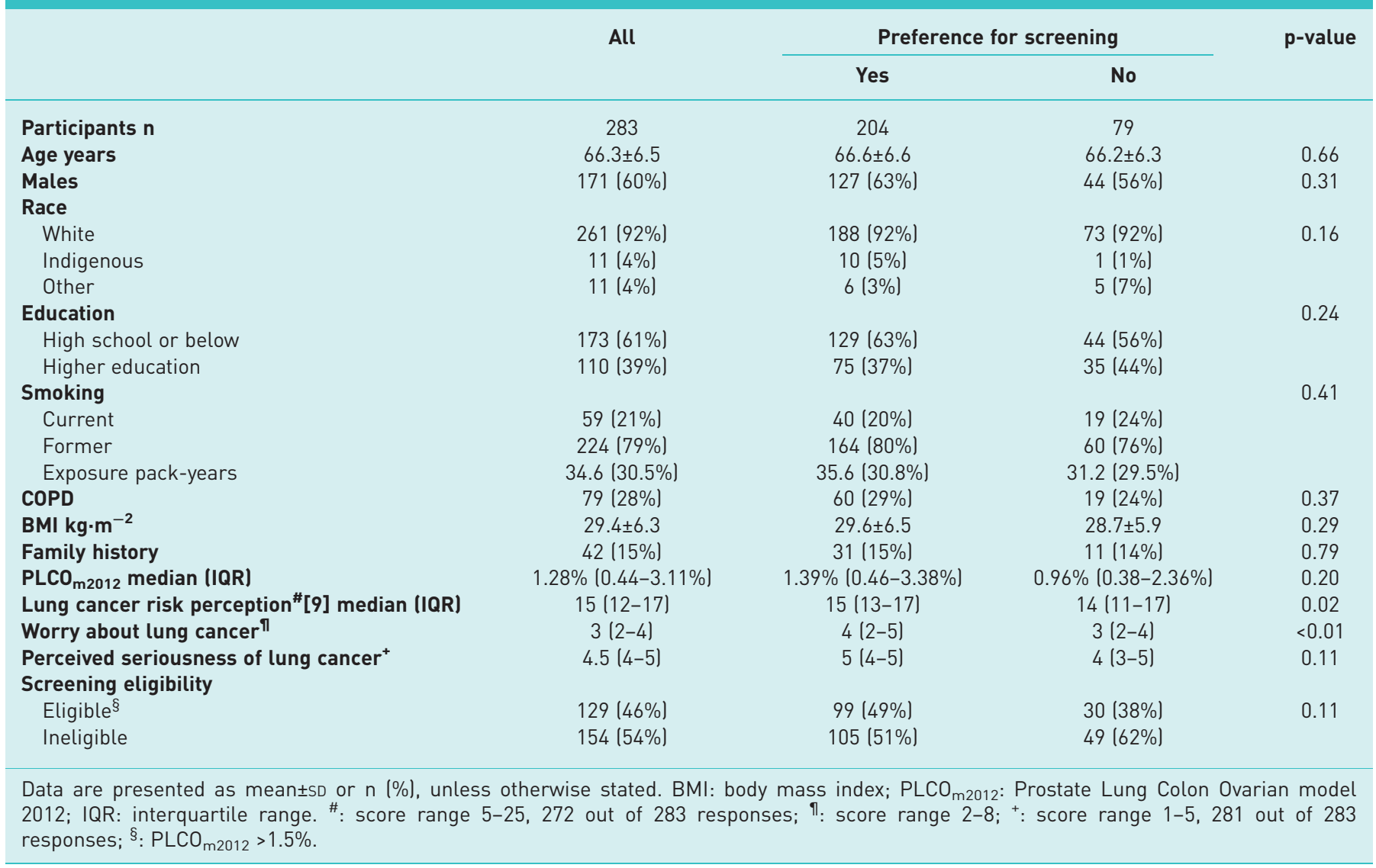




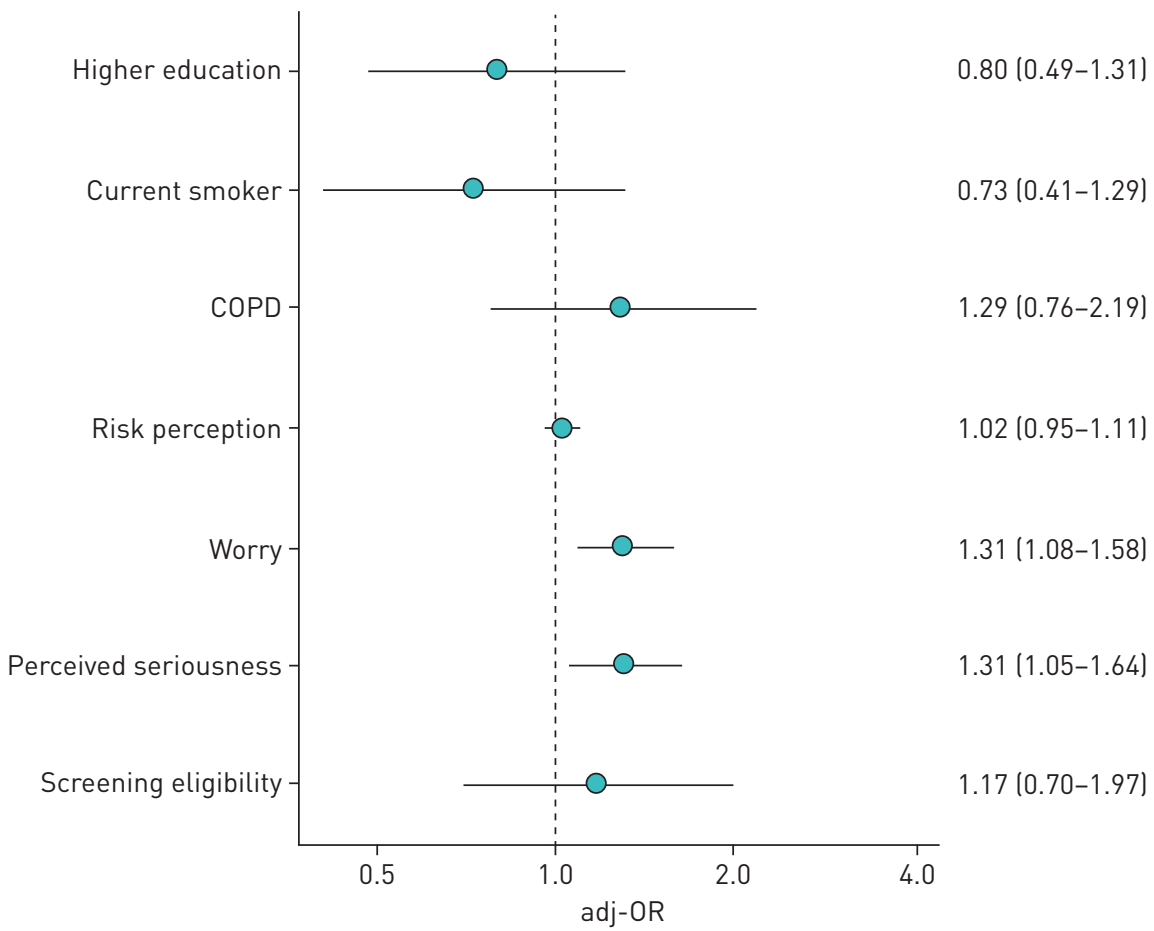

FIGURE 1 Adjusted-proportional odds ratios (adj-ORs) with 95\% confidence intervals from the multivariate ordinal logistic regression model to identify factors associated with higher preference for screening. COPD was self reported.

have lung cancer. Practical barriers, such as travel or opportunity costs from work or caring duties, were less important while concerns of radiation exposure and overdiagnosis were rated as more important (table 2).

\section{Discussion}

In this study, overall preference for screening was high, and worry about lung cancer and perceived seriousness of a lung cancer diagnosis were associated with a preference for participation in lung cancer

\section{TABLE 2 Comparison between perceived drivers and barriers to undergoing screening}

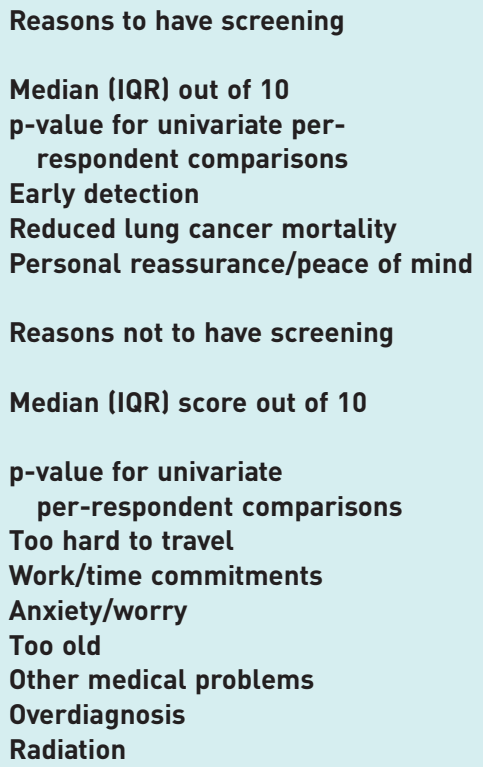

Early detection

$9(6-10)$

Early detection

$<0.0001$

$<0.0001$

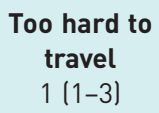

Too hard to travel

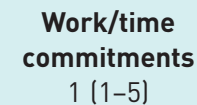

$1(1-5)$

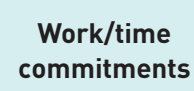

0.23

0.024

0.0002

0.0004

$<0.0001$

$<0.0001$
Reduced lung cancer mortality

$8(5-10)$

Reduced lung cancer mortality

0.54
IQR: interquartile range. Bold type indicates significant difference using Bonferroni-corrected $p$-value of 0.0023.
Personal reassurance/peace of mind

$8(5-10)$

Personal reassurance/peace of mind

$\begin{array}{cc}\text { Anxiety/ } & \text { Too } \\ \text { worry } & \text { old } \\ 2(1-5) & 2 \\ & (1-5) \\ \text { Anxiety/ } & \text { Too } \\ \text { worry } & \text { old }\end{array}$

Other medical

Overdiagnosis Radiation problems

$2(1-5) \quad 2(1-5) \quad 2(1-5)$

Other medical Overdiagnosis Radiation problems

0.27

0.21

0.11

0.6

0.36

0.69

0.3

0.59 
screening, while actual risk and eligibility for screening were not. A significant lung cancer screening implementation issue is to engage those at high risk, in whom the harm/benefit ratio is favourable, while at the same time minimising screening in those at low risk of lung cancer who have an unfavourable harm/benefit ratio. Previous qualitative studies have similarly described how emotional concerns about lung cancer or uncertainty can influence screening intentions of potential participants $[12,13]$. Understanding the factors that contribute to a preference for participation in lung cancer screening will better inform screening programmes in order to meet this challenge. Participants deemed opportunity costs related to screening and travel barriers least important. While our study, using validated measures, identified unique factors associated with screening preference, other factors not investigated, including distrust of the medical system, cost, and stigma about smoking and lung cancer, that may be relevant.

Half of those with a preference for screening were eligible based on their lung cancer risk. This high preference for participation is in keeping with other published studies describing attitudes towards lung cancer screening both in Australian and international populations, although these studies have not reported screening eligibility $[8,14]$. Screening of low-risk individuals leads to an unfavourable harm/ benefit ratio with large numbers needed to screen to prevent one lung cancer death, high rates of false positives, as well as the harms of overdiagnosis and psychological distress [11]. Conversely, almost a quarter of screening-eligible respondents did not have a preference for screening. Engaging with these individuals will continue to be an implementation issue.

There was a weak correlation between participants' lung cancer risk perception and objective lung cancer risk. Our study adds to other studies reporting contradictory data as to whether current smokers perceive their risk as greater than former smokers. Some researchers have reported that smokers overestimate the health risks of smoking, while others have reported accurate or underestimated perception of risk $[15,16]$. With poor accuracy of perception of risk combined with worry and seriousness of diagnosis being strongly associated with screening preference, attracting appropriate individuals to screening programmes remains difficult.

Our study provides a valuable insight into drivers for participation in lung cancer screening and is strengthened by the use of validated measures, but there are a number of limitations primarily related to selection, participation and nonresponse bias. We used a convenience sample of individuals attending outpatient clinics. By the nature of attending medical review, these individuals are inherently more engaged with their healthcare, and may be more likely to participate in screening and other healthcare interventions than current and former smokers in the general population. Furthermore, participants had a higher median lung cancer risk than previously reported in the general Australian population [2]. The study sample size was limited by feasibility issues leading to reduced explanatory power. While our outcome of self-reported screening preference is important, it has not been shown to correlate with screening behaviour or uptake, and the primary outcome may refer to a willingness to adhere to a screening recommendation rather than preference per se. The component of the questionnaire evaluating drivers and barriers to screening has not undergone formal validation, and the differences in median responses are clinically small, which may limit interpretation and/or generalisability. Nonetheless, our findings reveal important insights into the factors that are likely to drive participation in lung cancer screening and warrant further evaluation in population-based surveys. Further research into strategies to reduce worry in those at low risk of developing lung cancer is required so that screening programmes are able to successfully target those most likely to benefit.

\section{Conclusion}

Most current and former smokers would prefer to undergo screening if offered. Worry and perceived seriousness of a lung cancer diagnosis are strongly associated with the desire to participate in lung cancer screening, whilst there was no association with actual risk or screening eligibility. With the preference for participation in screening being high but similar between eligible and ineligible individuals, engaging with high-risk individuals while reducing inappropriate screening of low-risk individuals is a significant implementation challenge.

Acknowledgements: The authors would like to acknowledge and thank the administrative staff at St John of God Midland Hospital, and Shane Zaw who helped to distribute the survey and the survey participants for their time and contribution.

Conflict of interest: K. See has nothing to disclose. R. Manser has nothing to disclose. E.R. Park has nothing to disclose. D. Steinfort has no disclosures to declare B. King has nothing to disclose. F. Piccolo has nothing to disclose. D. Manners reports a speaking honorarium from AstraZeneca outside the submitted work. 


\section{References}

1 Moyer VA. Screening for lung cancer: U.S. preventive services task force recommendation statement. Ann Intern Med 2014; 160: 330-338.

2 Weber M, Yap S, Goldsbury D, et al. Identifying high risk individuals for targeted lung cancer screening: Independent validation of the $\mathrm{PLCO}_{\mathrm{m} 2012}$ risk prediction tool. Int J Cancer 2017; 141: 242-253.

3 Jemal A, Fedewa SA. Lung cancer screening with low-dose computed tomography in the United States-2010 to 2015. JAMA Oncol 2017; 3: 1278-1281.

4 Huo J, Shen C, Volk RJ, et al. Use of CT and chest radiography for lung cancer screening before and after publication of screening guidelines: intended and unintended uptake. JAMA Intern Med 2017; 177: $439-441$.

5 Manners D, Wilcox H, McWilliams A, et al. Current lung cancer screening practice amongst general practitioners in Western Australia: a cross-sectional study. Intern Med J 2018; 48: 78-80.

6 Canadian Task Force on Preventive Health Care. Recommendations on screening for lung cancer. CMAJ 2016 188: 425-432.

7 Federal Department of Health Standing Committee on Screening. Position Statement: Lung Cancer Screening using Low-Dose Computed Tomography. www.cancerscreening.gov.au/internet/screening/publishing.nsf/Content/ lung-cancer-screening. Date last updated: 6 November 2019. Date last accessed: 6 November 62019.

8 Flynn AE, Peters MJ, Morgan LC. Attitudes towards lung cancer screening in an Australian high-risk population Lung Cancer Int 2013; 789057.

9 Tanner NT, Egede LE, Shamblin C, et al. Attitudes and beliefs toward lung cancer screening among US veterans. Chest 2013; 144: 1783-1787.

10 Park ER, Ostroff JS, Rakowski W, et al. Risk perceptions among participants undergoing lung cancer screening: baseline results from the National Lung Screening Trial. Ann Behav Med 2009; 37: 268-279.

11 Tammemagi MC, Church TR, Hocking WG, et al. Evaluation of the lung cancer risks at which to screen everand never-smokers: screening rules applied to the PLCO and NLST cohorts. PLoS Med 2014; 11: e1001764.

12 Schapira MM, Aggarwal C, Akers S, et al. How patients view lung cancer screening. the role of uncertainty in medical decision making. Ann Am Thorac Soc 2016; 13: 1969-1976.

13 Lowenstein M, Vijayaraghavan M, Burke NJ, et al. Real-world lung cancer screening decision-making: Barriers and facilitators. Lung Cancer 2019; 133: 32-37.

14 Silvestri GA, Nietert PJ, Zoller J, et al. Attitudes towards screening for lung cancer among smokers and their non-smoking counterparts. Thorax 2007; 62: 126-130.

15 Park ER, Streck JM, Gareen IF, et al. A qualitative study of lung cancer risk perceptions and smoking beliefs among national lung screening trial participants. Nicotine Tob Res 2014; 16: 166-173.

16 Persoskie A, Mao Q, Chou WY, et al. Absolute and comparative cancer risk perceptions among smokers in two cities in China. Nicotine Tob Res 2014; 16: 899-903. 